\author{
Revista de Psicología Vol. 34 (1), 2016 (ISSN 0254-9247) \\ http://dx.doi.org/10.18800/psico.201601.008
}

\title{
Identificación colectiva y bienestar en una comunidad rural de la costa norte del Perú
}

\author{
Agustín Espinosa ${ }^{1}$, Silvana Freire ${ }^{2}$ y Jimena Ferrándiz ${ }^{3}$ \\ Pontificia Universidad Católica del Perú
}

\begin{abstract}
Este estudio examina las relaciones entre la identificación colectiva con una comunidad rural y las expresiones subjetiva, psicológica y social del bienestar. Estas variables fueron analizadas en 80 pobladores de la comunidad. A través de un modelo estructural se observa que el autoestereotipo positivo general incide positivamente sobre la autoestima colectiva, mientras que el autoestereotipo de baja eficacia, atenúa la misma. Por su parte la autoestima colectiva incrementa el grado de identificación. Los componentes autoestereotípicos presentan relaciones de influencia con distintas expresiones del bienestar. Así, el autoestereotipo de baja eficacia incide negativamente en el bienestar social y el autoestereotipo de corrupción influye negativamente en el bienestar psicológico. Finalmente, se observa que el bienestar social influye en el bienestar psicológico, y este en el bienestar subjetivo.

Palabras clave: comunidad rural, bienestar psicológico, bienestar social, bienestar subjetivo, identidad colectiva.
\end{abstract}

\section{Collective identification and well-being in a community from the Peruvian northern coast}

This study examines the association between collective identification with a rural community from the northern coast of Peru, and the subjective, psychological and social expressions of

1 Doctor en Psicología por la Universidad del País Vasco, Espańa y docente asociado del Departamento de Psicología de la Pontificia Universidad Católica del Perú. Coordinador del Grupo de Psicología Política de la PUCP. Dirección postal: Av. Universitaria 1801, Lima 32. Contacto: agustin.espinosa@pucp.edu.pe

2 Licenciada en Psicología con mención en Psicología Social por la Pontificia Universidad Católica del Perú y profesional en el Ministerio de la Mujer y Poblaciones Vulnerables. Es miembro del Grupo de Psicología Política de la PUCP. Dirección postal: Av. Grau 915, Lima 04. Contacto: sfreire@grade.org.pe

3 Licenciada en Psicología con mención en Psicología Social por la Pontificia Universidad Católica del Perú. Profesional en el Ministerio de Educación. Es miembro del Grupo de Psicología de la PUCP. Dirección postal: Av. Álvarez Calderón 336 San Isidro. Contacto: jimena.ferrandiz@pucp.edu.pe 
well-being. These variables were analyzed in a sample of 80 community residents. Results show significant associations between some components of collective identification and some expressions of well-being. A path analysis suggest that the general positive self-stereotype has a positive effect on collective self-esteem, while the self-stereotype of low efficacy reduces it. Meanwhile, collective self-esteem increases the degree of identification. Self-stereotype components influence different expressions of well-being. Thus, self-stereotype of low efficacy negatively affects social well-being, and self-stereotype of corruption negatively affects psychological well-being. Finally, social well-being influences psychological wellbeing, which in turn influences subjective well-being.

Keywords: collective identity, psychological well-being, rural community, social well-being, subjective well-being.

\section{Identificaçáo coletiva e bem-estar em uma comunidade rural da costa norte do Peru}

Este estudo examinou a relação entre a identificação coletiva em uma comunidade rural e as expressôes do bem-estar subjetivo, psicológico e social. Estas variáveis foram analisadas em 80 moradores da comunidade. Através de um modelo estrutural mostra-se que o auto-estereótipo positivo geral aumenta a autoestima coletiva, enquanto o auto-estereótipo de baixa eficácia, diminui a mesma. Enquanto isso, a autoestima coletiva aumenta o grau de identificação com a comunidade. As dimensôes do auto-estereótipo tem relaçóes de influência com as diferentes expressōes do bem-estar. Assim, o auto-estereótipo de baixa eficácia afeta negativamente o bem-estar social e o auto-estereótipo de corrupção afeta negativamente o bem-estar psicológico. Finalmente, observa-se que o bem-estar social influencia o bem-estar psicológico, e este influencia o bem-estar subjetivo.

Palavras-chave: comunidade rural, bem-estar psicológico, bem-estar social, bem-estar subjetivo e identidade coletiva. 
Desde una aproximación psicológica, la identidad es entendida como una conceptualización subjetiva sobre uno mismo, la cual es construida a partir de la interacción de distintos procesos cognitivos, afectivos y sociales, insertos en escenarios culturales y contextos locales específicos (Tajfel, 1984; Vignoles, Regalia, Manzi, Golledge \& Scabini, 2006). El estudio de los aspectos colectivos de la identificación se aborda desde el concepto de identidad social, entendido como "aquella parte del autoconcepto de un individuo que deriva del conocimiento de su pertenencia a un grupo social junto con el significado valorativo y emocional asociado a dicha pertenencia” (Tajfel, 1984, p. 292). Esta aproximación postula que la pertenencia a un grupo representa un estado psicológico (Tajfel, 1982) e implica una evaluación y valoración del sí mismo a partir de los atributos del grupo al cual uno pertenece (Tajfel \& Turner, 2001).

Diversos autores han establecido que la identidad social influye en el bienestar al facilitar la adaptación de los individuos a su medio (Haslam, Jetten, Postmes \& Haslam, 2009; Reicher \& Haslam, 2006; Vignoles et al., 2006). Al respecto, la identidad social suele ser fuente de autoestima personal (Abrams \& Hogg, 1990) y dadas las estrechas relaciones entre la autoestima personal y el bienestar subjetivo, algunas propuestas conceptuales y posteriores hallazgos empíricos sugieren que este tipo de identificación también debería tener efectos positivos en la expresión del bienestar subjetivo, psicológico y social (Espinosa \& Tapia, 2011; Haslam et al., 2009; Lyubomirsky, Tkach \& DiMatteo, 2006).

Haslam y colaboradores (2009), Haslam, O’Brien, Jetten, Vordemal y Penna (2005), y Basabe, Páez, Aierdi y Jiménez (2009) señalan que la identidad social posee un impacto positivo en el bienestar subjetivo, cuando a partir de esta se facilita el establecimiento de redes de soporte social entre los miembros de los grupos a los que una persona pertenece. La satisfacción con tales redes facilita la capacidad para trabajar 
coordinadamente con otros, reduciendo las consecuencias negativas de eventos potencialmente estresantes, al proveer a los individuos de un sentido de aceptación y autovaloración, afiliación y contacto con otros, ayuda concreta, y mayor dominio del entorno (Basabe et al., 2009; Bobowik, Basabe \& Páez, en prensa). En ese sentido, la identificación social, expresada a partir de la membresía y pertenencia a un grupo, también genera repercusiones positivas para el bienestar psicológico de los individuos, promoviendo sentimientos de distinción, eficacia y éxito (Haslam et al., 2009), y ayudándolos a ajustarse a las transiciones que experimentan a lo largo de sus vidas (Bobowil et al., en prensa; Reicher \& Haslam, 2006).

En Perú, Espinosa y Tapia (2011), y en México, Laca, Mejía y Yáñez, (2010) encuentran en sus estudios una relación positiva de la identificación nacional con las distintas dimensiones del bienestar social, expresado este último en un sentido de integración, una mayor aceptación social y confianza interpersonal, una mayor sensación de contribución con la sociedad, una percepción de un buen funcionamiento institucional y una mayor comprensión del sistema.

Ante este escenario conceptual, resulta de interés indagar sobre la forma en que se constituye la identidad colectiva en una comunidad rural de la costa norte del Perú, además de analizar la relación que los componentes de dicha identificación tienen con las expresiones del bienestar subjetivo, psicológico y social en los pobladores de la misma. Lo anterior se justifica pues en los últimos 40 ańos, los centros poblados rurales de la costa norte del Perú han afrontado una serie de acontecimientos sociales y políticos que progresivamente han debilitado los procesos cooperativos y han deteriorado el tejido social en dichas comunidades. Esto ha generado, entre sus habitantes, la instauración de una serie de prácticas económicas y sociales de corte individualista (Balbuena, 2013; La Barrera, Espinosa, Cueto \& Ferrándiz, 2012), que parecen tener un efecto negativo en los procesos de cohesión social y participación comunitaria (Montero, 2010), afectando por consiguiente los procesos de identificación colectiva y bienestar de sus pobladores (Espinosa, Ferrándiz, Cueto \& Pain, 2013; La Barrera et al., 2012). 


\section{Método}

\section{Participantes}

Los participantes del estudio son habitantes de un centro poblado menor ubicado en la costa norte del Perú, en la provincia de Chepén, Región de La Libertad. Esta comunidad cuenta con alrededor de 200 hogares cuyas economías dependen principalmente de actividades agrícolas. La muestra estuvo compuesta por 31 hombres y 49 mujeres entre los 17 y 71 años de edad $(M=38.99, D E=13.92)$, todos ellos residentes en la comunidad.

\section{Medidas e instrumentos de medición}

a. Grado de identificación con la comunidad (Espinosa, 2011): se utilizó un ítem que mide el grado de identificación que posee un individuo en relación con su comunidad a través de la siguiente pregunta: ¿Cuál es su grado de identificación con la comunidad? La escala de respuestas va del 1 al 5, donde 1 es "Nada" y 5 es "Total". La relación significativa de este ítem con indicadores identitarios de autoestima y autoestereotipia da cuenta de su validez de criterio concurrente y validez de constructo convergente en diferentes contextos sociales (cf., Beramendi, 2013; Espinosa et al., 2013; Espinosa, 2011; Genna \& Espinosa, 2012).

b. Escala de Autoestima Colectiva (Luhtanen \& Crocker, 1992): esta medida es una traducción al español y adaptación al Perú de la sub escala de autoestima colectiva privada de Luhtanen y Crocker (Espinosa, 2011). Posee cuatro ítems que evalúan la relación afectiva de los participantes respecto a su identificación con la comunidad. Las respuestas van del 1 al 5 en una escala tipo Likert, donde 1 es "Totalmente en desacuerdo" y 5 es "Totalmente de acuerdo". Para este estudio la escala mostró una buena consistencia interna $(\alpha=.76)$. La escala ha sido aplicada con éxito para el análisis de diversas categorías sociales y étnicas en el contexto peruano. Además se relaciona consistentemente con un conjunto de variables psicosociales, lo que da cuenta de su validez de criterio concurrente y convergente (cf. Espinosa, 2011; Espinosa et al., 2013). 
c. Escala de Autoestereotipia Colectiva (Espinosa, 2011): esta escala incluye una lista de 24 adjetivos que describen cómo son los pobladores de la comunidad. Para cada adjetivo, se presenta una escala de tipo Likert que va del 1 al 5, donde 1 es "Totalmente en desacuerdo" y 5 es "Totalmente de acuerdo". Los 24 adjetivos se sometieron a un análisis factorial de tipo exploratorio que dio como resultado una estructura factorial (KMO = .760) de cuatro factores denominados: "baja eficacia", "imagen positiva general", "corruptos" y "expresividad positiva".

La Tabla 1 describe la agrupación de los atributos asociados a cada dimensión del autoestereotipo colectivo de la comunidad, y presenta las cargas factoriales respectivas, así como los niveles de varianza explicada y de consistencia interna para cada dimensión.

La estructura factorial obtenida replica los contenidos conseguidos en otros estudios sobre identidades colectivas realizadas en el contexto peruano (cf., Espinosa, 2011). A pesar de la variabilidad de los resultados producidas por el análisis factorial exploratorio, la consistencia de las dimensiones obtenidas con otros estudios da cuenta de la validez de la escala.

a. Escala de Satisfacción con la vida (Diener, Emons, Larsen \& Griffin, 1985): la escala consta de cinco ítems que evalúan el nivel de satisfacción con la vida de los individuos de manera global. La escala de respuestas que va del 1 al 7, donde 1 es "No, en absoluto" y 7 es "Sí, totalmente". Se aplicó la versión revisada por Pavot y Diener (1993), en su versión validada al Perú (Martínez, 2004). Para el presente estudio la escala obtuvo un nivel de consistencia interna aceptable $(\alpha=.54)$.

b. Escala de Bienestar Psicológico (Ryff, 1989; Díaz et al., 2006): la escala evalúa el bienestar a través de elementos como la autoaceptación, la capacidad de establecer relaciones positivas con otras personas, las oportunidades personales de tener crecimiento personal, la sensación de dominar el entorno, la autonomía y el hecho de tener un propósito en la propia vida. A través de un estudio piloto en población urbanomarginal de Lima, se adaptó la versión original de 39 ítems a una versión reducida de 19 ítems que fuera, en términos de respuesta, más accesible a los habitantes del centro poblado donde se desarrolló el estudio. 


\section{Tabla 1}

Análisis Factorial Exploratorio del Autoestereotipo Colectivo

\begin{tabular}{|c|c|c|c|c|}
\hline & \multicolumn{4}{|c|}{ Componentes } \\
\hline & Baja eficacia & $\begin{array}{l}\text { Imagen positiva } \\
\text { general }\end{array}$ & Corruptos & $\begin{array}{c}\text { Expresividad } \\
\text { positiva }\end{array}$ \\
\hline Incumplidos & .73 & & & \\
\hline Atrasados & .68 & & & \\
\hline Ociosos & .68 & & & \\
\hline Incapaces & .68 & & & \\
\hline Mentirosos & .66 & & & \\
\hline Conformistas & .70 & & & \\
\hline Individualistas & .45 & & & \\
\hline Exitosos & & .74 & & \\
\hline Valientes & & .74 & & \\
\hline De confianza & & .80 & & \\
\hline Cumplidos & & .69 & & \\
\hline Honestos & & .56 & & \\
\hline Trabajadores & & .56 & & \\
\hline Capaces & & .54 & & \\
\hline No confiables & & -.46 & & \\
\hline Fracasados & & & .71 & \\
\hline Corruptos & & & .64 & \\
\hline Honrados & & & -.58 & \\
\hline Alegres & & & & .79 \\
\hline Tristes & & & & -.55 \\
\hline $\begin{array}{l}\text { Consistencia } \\
\text { interna }\end{array}$ & .81 & .78 & .54 & .48 \\
\hline $\begin{array}{l}\text { Varianza } \\
\text { explicada (\%) }\end{array}$ & 24.03 & 15.63 & 8.98 & 6.49 \\
\hline
\end{tabular}

Nota. Método de extracción: Análisis de Componentes Principales. Método de rotación: Varimax con Normalización de Kaiser. La rotación convergió en 8 iteraciones. 
El instrumento emplea una escala de respuesta de tipo Likert del 1 al 6, donde 1 es "Fuertemente en desacuerdo" y 6 es "Fuertemente de acuerdo". A pesar que la escala mide el bienestar psicológico a partir de seis dimensiones, para el presente estudio sólo se utilizó la puntuación general de la misma, cuya consistencia interna es buena $(\alpha=.75)$.

c. Escala de Bienestar Social (Keyes, 1998; Blanco \& Díaz, 2005): mide la evaluación global del propio individuo acerca de su circunstancia y funcionamiento en la sociedad (Keyes, 1998). A través de un estudio piloto se adaptó la versión original de 33 ítems a una versión reducida de 15, que emplea una escala Likert del 1 al 5, donde 1 es "Fuertemente en desacuerdo" y 5 es "Fuertemente de acuerdo". A pesar de que la escala evalúa el bienestar social a partir de 5 dimensiones, para el presente estudio se trabajará con la puntuación general de la misma, cuya consistencia interna es buena $(\alpha=.74)$.

\section{Procedimiento y procesamiento de información}

La medición de las variables del estudio se hizo a partir de una serie de visitas al centro poblado con la ayuda de un equipo de investigación. Se realizaron convocatorias para que los pobladores asistiesen a reuniones previamente pactadas y en algunos casos resultó necesario realizar aplicaciones individuales, buscando a los pobladores de casa en casa. Los cuestionarios fueron llenados de manera anónima, contando con el consentimiento informado de los participantes. Si bien el instrumento era autoaplicado, el equipo de investigación estuvo presente para resolver las dudas de los participantes.

La información recogida durante el trabajo de campo fue digitada y analizada en el paquete estadístico SPSS versión 18. Asimismo, se realizó un análisis estadístico multivariado, proponiendo un path analysis - a través del programa de estimación AMOS 20 (Analysis of Moment Structures), para analizar la relación entre las variables estudiadas. 


\section{Resultados}

La puntuación media para las variables que conforman el indicador de Grado de identificación con la comunidad es de $M=3.39$, $D E=1.04$, mientras que la puntuación en la medida de autoestima colectiva es de $M=4.24, D E=.77$. Ambas puntuaciones se encuentran por encima del punto medio de las escalas utilizadas (3). En relación con la escala de Autoestereotipia Colectiva, a nivel descriptivo, los participantes del estudio presentan un mayor acuerdo con los atributos autoestereotípicos positivos. Ello se evidencia en la percepción de los factores de Expresividad positiva, $(M=4.09, D E=.74)$ e Imagen positiva general $(M=3.64, D E=.67)$, en comparación a los factores de Baja eficacia $(M=2.88, D E=.79)$ y Corruptos $(M=2.08, D E=.77)$.

Respecto a las puntuaciones medias de las medidas de bienestar, el Bienestar Subjetivo presenta una media de $M=4.11, D E=1.14$, el Bienestar Psicológico una media de $M=4.57, D E=.55$, y el Bienestar Social una media de $M=3.45, D E=.56$.

Luego, se procesaron correlaciones tipo Pearson para analizar las relaciones entre los componentes de la identidad social y las tres expresiones de bienestar evaluadas.

Los resultados de la tabla 2 muestran relaciones estadísticas significativas de efectos moderados a altos $|.19 \leq \mathrm{r} \leq .64|$, la mayoría de ellos por encima de la mediana del tamaño del efecto $|\mathrm{r}=.21|$ de la tradición investigativa en psicología social (cf., Richard, Bond \& Stokes-Zoota, 2003). De manera específica, se aprecia que el grado de identificación con la comunidad no se relaciona directamente con ninguno de los tipos de bienestar medidos. Sin embargo, la autoestima colectiva y las cuatro dimensiones autoestereotípicas se asocian consistentemente con el bienestar psicológico y el bienestar social. 


\section{Tabla 2}

Correlaciones entre los componentes de la identificación social, el bienestar subjetivo, bienestar psicológico y bienestar social

\begin{tabular}{|c|c|c|c|c|c|c|c|c|c|}
\hline Variables & 1 & 2 & 3 & 4 & 5 & 6 & 7 & 8 & 9 \\
\hline $\begin{array}{l}\text { Grado de } \\
\text { identificación }\end{array}$ & - & & & & & & & & \\
\hline $\begin{array}{l}\text { Autoestima } \\
\text { colectiva }\end{array}$ & $.56^{* * *}$ & - & & & & & & & \\
\hline Baja eficacia & -.12 & $-.37^{* *}$ & - & & & & & & \\
\hline $\begin{array}{l}\text { Imagen positiva } \\
\text { general }\end{array}$ & .18 & $.29^{* *}$ & $-.23^{*}$ & - & & & & & \\
\hline Corruptos & $-.25^{*}$ & $-.30 * *$ & $.37^{* *}$ & $-.34^{* *}$ & - & & & & \\
\hline $\begin{array}{l}\text { Expresividad } \\
\text { positiva }\end{array}$ & .15 & .17 & $-.28^{*}$ & .14 & $-.41^{* * *}$ & - & & & \\
\hline $\begin{array}{l}\text { Bienestar } \\
\text { subjetivo }\end{array}$ & .14 & .13 & -.09 & .10 & -.13 & .11 & - & & \\
\hline $\begin{array}{l}\text { Bienestar } \\
\text { psicológico }\end{array}$ & .18 & $.22^{*}$ & $-.36^{* *}$ & $.20+$ & $-.29^{* *}$ & $.30^{* *}$ & $.26^{*}$ & - & \\
\hline Bienestar social & .11 & $.27^{*}$ & $-.34^{* *}$ & $.28^{*}$ & $-.19+$ & $.21+$ & .17 & $.64^{* * *}$ & - \\
\hline
\end{tabular}

Nota: ${ }^{* * *} p<.001,{ }^{* *} p<.01,{ }^{*} p<.05,+1 . n=80$.

En ese sentido, se propone analizar en un modelo más complejo las posibles relaciones de influencia entre las variables antes descritas. Para tal fin, con sustento en la revisión conceptual y en las relaciones empíricamente aquí obtenidas, se ha propuesto un modelo de análisis de ruta que sugiere que en un primer paso, los componentes autoestereotípicos influyen en la autoestima colectiva. De manera específica, los componentes positivos la incrementan y los negativos la mitigan. En un segundo momento, la autoestima colectiva influiría en el grado de identificación, y este a su vez influiría directamente en el bienestar social, el cual afectaría positivamente en las expresiones psicológica y subjetiva del bienestar, tal como se muestra en la siguiente figura. 


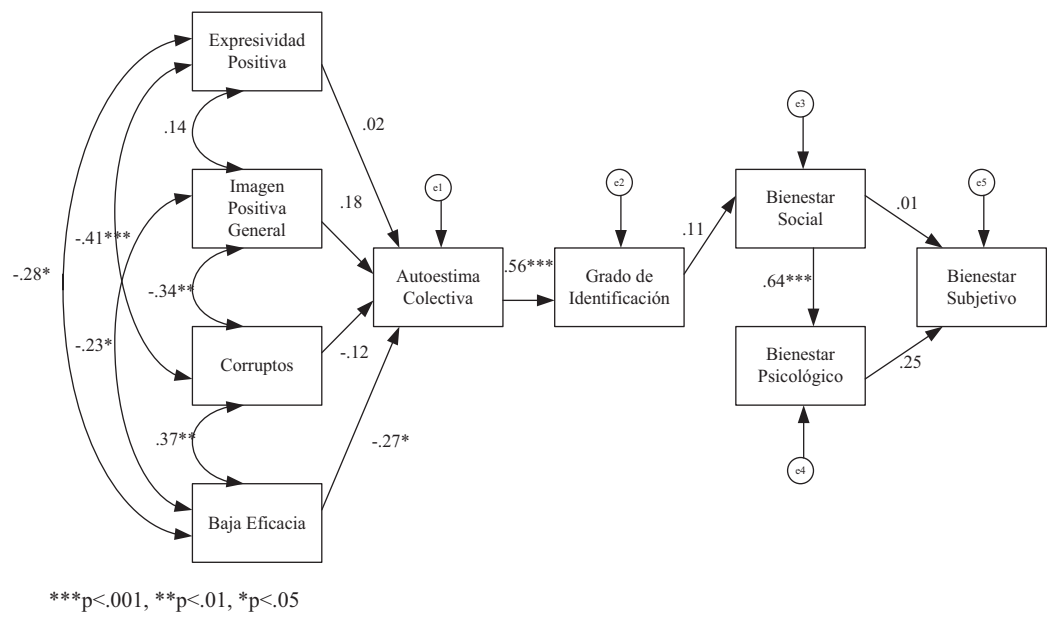

Figura 1. Análisis de ruta de las relaciones entre los componentes de la identidad social y las expresiones del bienestar (modelo hipotético).

De acuerdo con el criterio establecido por distintos autores (cf., Kline, 2005; Schumacker \& Lomax, 2004), el modelo hipotético propuesto, presenta un buen nivel de ajuste de los datos y bajos niveles de error (ver Tabla 3). Sin embargo, muchos de los valores de beta reportados en el modelo no resultan significativos, por lo que se analizan posibles soluciones alternativas resultando elegida, por sus buenos niveles de ajuste, bajos niveles de error y significación de los valores de beta y r (ver Tabla 3), un modelo alternativo que sugiere que el autoestereotipo positivo general incide positivamente sobre la autoestima colectiva, mientras el autoestereotipo de baja eficacia, atenúa la misma. Por su parte la autoestima colectiva incrementa el grado de identificación. Asimismo, el autoestereotipo de baja eficacia incide negativamente en el bienestar social y el autoestereotipo de corrupción produce el mismo efecto en el bienestar psicológico. No se aprecia una relación directa de la autoestima o del grado de identificación en ningún tipo de bienestar. Las relaciones de influencia entre las dimensiones 
del bienestar van desde el bienestar social al bienestar psicológico, y de este al bienestar subjetivo.

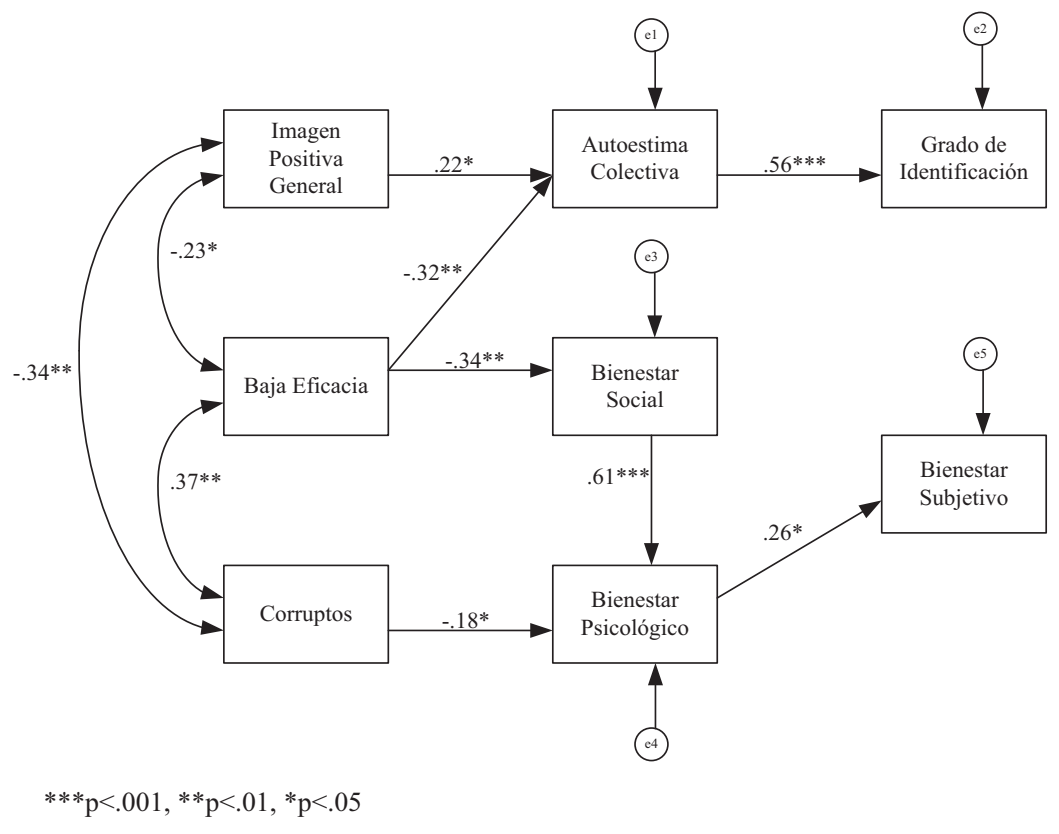

Figura 2. Análisis de ruta de las relaciones entre los componentes de la identidad social y las expresiones del bienestar (modelo alternativo)

\section{Tabla 3}

Niveles de ajuste y error de los modelos de relaciones de los componentes de la identidad social y las expresiones de bienestar

\begin{tabular}{lcccccc}
\hline Modelos & $\chi^{2} / \mathrm{gl}$ & GFI & AGFI & CFI & RMR & RMSEA \\
\hline M. Hipotético & 1.30 & .933 & .856 & .949 & .064 & .062 \\
M. Alternativo & .74 & .961 & .922 & 1.00 & .046 & .001 \\
\hline
\end{tabular}




\section{Discusión}

De manera general, los resultados del presente estudio corroboran que algunos componentes de la identificación colectiva se asocian a las distintas expresiones del bienestar (Espinosa \& Tapia, 2011; Haslam et al., 2009; Reicher \& Haslam, 2006; Vignoles et al., 2006). Sin embargo, se aprecian ciertas particularidades que son importantes de resaltar. En primer lugar, las influencias de los componentes de la identidad en las distintas expresiones de bienestar no están vinculadas directamente con el grado de identificación, ni con los niveles de autoestima, sino con las representaciones autoestereotípicas predominantes en la comunidad. De manera específica, son los contenidos autoestereotípicos de baja eficacia y corrupción los que inciden negativamente en el bienestar social y en el bienestar psicológico respectivamente. Adicionalmente, contra lo esperado no se aprecian influencias directas de ningún componente de la identificación colectiva con el bienestar subjetivo.

Estudios previos en el contexto peruano que analizaban la identificación con el endogrupo nacional encontraron que el grado de identificación y la autoestima colectiva se relacionaban directamente con la expresiones sociales y subjetivas del bienestar (cf., Espinosa \& Tapia, 2011). Sin embargo, estos resultados no se replicaron claramente en el presente estudio, lo que sugiere que no todas las categorías sociales con la que un individuo podría identificarse presentan el mismo efecto en el bienestar. Lo que si resulta necesario atender con miras a mejorar la calidad de vida de los habitantes de la comunidad son las condiciones sociales y políticas que a lo largo de la historia de la misma han incidido en la imagen autoestereotípica negativa de sus miembros, mitigando la acción colectiva y debilitando el tejido social (e.g. La Barrera et al., 2012; Espinosa, Ferrándiz et al., 2013; Freire, 2013). Lo anterior es consistente con lo propuesto por Van Vugt y Hart (2004), quienes afirman que la identificación social funciona como un mecanismo de cohesión basado en las expectativas positivas que un individuo tenga sobre los miembros del endogrupo. En el caso 
de la comunidad de estudio, aunque los niveles de identificación y valoración de pertenencia a la misma han sido elevados, se observan niveles de desconfianza anclados en las percepciones y experiencias negativas que se han ido dando entre conciudadanos (e.g. Espinosa, Ferrándiz et al., 2013; Freire, 2013; La Barrera et al., 2012).

Por una parte, el autoestereotipo de corrupción parece haberse constituido sobre la base de las experiencias constantes de estafas que los miembros de la comunidad han experimentado a lo largo de los últimos años. El panorama se agrava, porque si bien las estafas han sido realizadas principalmente por agentes externos a la comunidad; finalmente, muchos miembros de la misma han sido beneficiarios políticos o receptores de prebendas producidas por esta situación, en desmedro de otros. Esto ha generado las prácticas de producción y de funcionamiento social individualistas orientadas a lograr una supervivencia individual antes que el desarrollo colectivo (Balbuena, 2013; La Barrera et al., 2012). Este autoestereotipo afecta directamente el bienestar psicológico, pues parece haberse naturalizado la percepción de la comunidad como un espacio donde el desarrollo depende de uno mismo, y donde las condiciones para que esto ocurra son limitadas, lo que finalmente podría incidir de manera negativa en la percepción de dominio del entorno.

El autoestereotipo de baja eficacia, también parece estar relacionado con la historia de la comunidad, en la medida en que la misma ha ido dirigiéndose a un empobrecimiento progresivo (Balbuena, 2013). Esta baja eficacia colectiva afecta el bienestar social, pues surge una sensación de falta de predictibilidad sobre el funcionamiento social de la comunidad hacia el futuro. Lo que se vincula por una parte a la inestabilidad propia de la actividad agrícola y a la sensación de que el colectivo no estará presente para una acción colectiva organizada ante situaciones de crisis, sino que la cohesión se debilitará aún más en estas circunstancias.

De manera general, se observa que existe una visión negativa del funcionamiento de la organización comunitaria. La visión negativa institucional predominante en la comunidad, no solo está circunscrita 
a un sistema de control social ineficiente, sino que afecta las descripciones identitarias de los habitantes de la comunidad, encontrándose un alineamiento entre el sistema predominante en la comunidad y las representaciones de los habitantes que lo habitan (Beramendi \& Zubieta, 2013), lo que explica las influencias del funcionamiento de una sociedad en la identidad colectiva (Beramendi \& Zubieta, 2013; Espinosa, 2011).

La falta de influencia directa de los componentes de la identidad sobre el bienestar subjetivo parece explicarse por el hecho de que esta expresión del bienestar es preponderantemente personal (Espinosa \& Tapia, 2011). Sin embargo, una aproximación complementaria propone que para entender el funcionamiento óptimo y la salud mental de las personas a partir de un sentido de integración con el endogrupo y la adaptación al entorno, es necesario considerar previamente las perspectivas del bienestar social y psicológico, ya que las condiciones sociales sobre las que se constituyen los afectos positivos y los juicios globales de satisfacción con la propia vida dependen de condiciones psicológicas previas de confianza interpersonal, de sensación de que la comunidad es un escenario que promueve el crecimiento personal y de que el funcionamiento social comunitario es positivo y comprensible. (Haslam et al., 2005; Haslam et al., 2009). En ese sentido, hasta que no se apuntalen o se mejoren las condiciones sociales que garanticen una convivencia claramente regulada y de respeto al interior de la comunidad, los aspectos identitarios positivos se verán mitigados y la calidad de vida de sus pobladores se verá indirecta y negativamente afectada.

\section{Referencias}

Abrams, D. \& Hogg, M.A. (1990). An introduction to the social identity approach. En D. Abrams \& M.A. Hogg (Eds.), Social Identity Theory: Constructive and Critical Advances (pp. 1-9). Hertfordshire: Harvester Wheatsheaf. 
Balbuena, A. (2013). Sentido de comunidad, bienestar y memoria colectiva en una comunidad rural de la costa norte peruana. Tesis de licenciatura en psicología social no publicada. Lima: Pontificia Universidad Católica del Perú.

Basabe, N., Páez, D., Aierdi, X. \& Jiménez-Aristizabal, A. (2009). Salud e Inmigración. Aculturación, bienestar subjetivo y calidad de vida. Zarautz: Ikuspegi

Beramendi, M. (2013). Percepción del sistema normativo, transgresión y sus correlatos psicosociales en Argentina. Tesis doctoral en psicología social no publicada. Buenos Aires: Universidad de Buenos Aires.

Beramendi, M. \& Zubieta, E. (2013). La identidad nacional y relaciones interpersonales en una cultura donde la norma es la transgresión. Psicologia Politica, 26(13), 165-177.

Blanco, A. \& Díaz, D. (2005). El bienestar social: su concepto y medición. Psicothema, 17(4), 582-589.

Bobowik, M., Basabe, N. \& Páez, D. (En prensa). The bright side of migration: hedonic, psychological, and social well-being in inmigrants in Spain. Social Science Research.

Díaz, D., Rodríguez-Carvajal, R., Blanco, A., Moreno-Jiménez, B., Gallardo, I., Valle, C. \& van Dierendonck, D. (2006). Adaptación española de las escalas de bienestar psicológico de Ryff. Psicothema, 18(3), 572-577.

Diener, E., Emmons, R., Larsen, R. \& Griffin, S. (1985). The Satisfaction With Life Scale. Journal of Personality Assessment, 49(1),71-75.http://dx.doi.org/10.1207/s15327752jpa4901_13

Espinosa, A. (2011). Estudios sobre identidad nacional en el Perú y sus correlatos psicológicos, sociales y culturales. Tesis doctoral en psicología social no publicada. San Sebastián: Universidad del País Vasco.

Espinosa, A., Ferrándiz, J., Cueto, R. M. \& Pain, O. (2013). Social identity and emotional climate in a rural community of Peru: an empirical study. Psicología \& Sociedade, 25(2), 321-330. http://dx.doi.org/10.1590/S0102-71822013000200009 
Espinosa, A. \& Tapia, G. (2011). Identidad nacional como fuente de bienestar subjetivo y social. Boletín de Psicología, 102, 71-88.

Freire, S. (2013). Identificación con el lugar, participación y clima emocional en una comunidad rural de la costa norte (Tesis de licenciatura en psicología social no publicada). Lima: Pontificia Universidad Católica del Perú.

Genna, K. \& Espinosa, A. (2012). Identidad, etnicidad y bienestar social en un contexto socialmente excluyente. Psicologia \& Sociedade, 24(1), 84-93. http://dx.doi.org/10.1590/S010271822012000100010

Haslam, S. A., Jetten, J, Postmes, T. \& Haslam, C. (2009). Social Identity, Health and Well- Being: An Emerging Agenda for Applied Psychology. Applied Psychology: an international review, 58(1), 1-23. http://dx.doi.org/10.1111/j.1464-0597.2008.00379.x

Haslam, S. A., O’brien, A., Jetten, J., Vordemal, K. \& Penna, S. (2005). Taking the strain: Social identity, social support, and the experience of stress. British Journal of Social Psychology, 44(3), 355-370. http://dx.doi.org/10.1348/014466605X37468

Keyes, C. (1998). Social Well-Being. Social Psychology Quarterly, 61(2), 121-140. http://dx.doi.org/10.2307/2787065

Kline, R. B. (2005). Principles and practice of structural equation modeling (2nd ed.). New York: Guilford.

La Barrera, P., Espinosa, A., Cueto, R. M. \& Ferrándiz, J. (2012). Aspectos psicológicos de los problemas de organización de base y su relación con dilemas sociales en una comunidad rural de la costa norte del Perú. Psicoperspectivas, 11(1), 82-107. http:// dx.doi.org/10.5027/psicoperspectivas-Vol11-Issue1-fulltext-184

Laca, F., Mejía, J. C. \& Yañez, C. (2010). Identidad mexicana e interés político: Predictores de bienestar social y anomia. Acta Universitaria, 20(2), 40-49.

Luhtanen, R. \& Crocker, J. (1992). A collective self-esteem scale: Self evaluation of one's identity. Personality and Social Psychology Bulletin, 18(3), 302-318. http://dx.doi.org/10.1177 /0146167292183006 
Lyubomirsky, S., Tkach, C. \& DiMatteo, M. M. (2006). What are the Differences between Happiness and Self-Esteem. Social Indicators Research, 78(3), 363-404. http://dx.doi.org/10.1007/ s1 1205-005-0213-y

Martínez, P. (2004). Perspectiva temporal futura y satisfacción con la vida a lo largo del ciclo vital. Revista de Psicología, 22(2), 215-252.

Mezulis, A. H., Abramson, L. Y., Hyde, J. S. \& Hankin, B. L. (2004). Is there a universal positivity bias in attributions? A meta-analytic review of individual, developmental and cultural differences in the self-serving attributional bias. Psychological Bulletin, 130(5), 711-747. http://dx.doi.org/10.1037/00332909.130.5.711

Montero, M. (2010). Unión de actores sociales, participación comunitaria y ética en la ejecución de políticas públicas. En A. E. Hincapié (Ed.), Sujetos políticos y acción comunitaria (pp. 45-66). Medellín: Universidad Pontificia Bolivariana.

Pavot, W. \& Diener, E. (1993). Review of the Satisfaction with Life Scale. Psychological Assessment, 5(2), 164-172. http://dx.doi. org/10.1037/1040-3590.5.2.164

Richard, F. D., Bond Jr., Charles F. \& Stokes-Zoota, J. J. (2003). One Hundred Years of Social Psychology Quantitatively Described. Review of General Psychology, 7(4), 331-63. http://dx.doi. org/10.1037/1089-2680.7.4.331

Reicher, S. \& Haslam, S. A. (2006). Tyranny revisited: Groups, psychological well- being and the health of societies. Psychologist, 19(3), 146-150.

Schumaker, R. E., \& Lomax, R. G. (2004). A beginner's guide to structural equation modeling (2nd ed.). Mahwah, NJ: Lawrence Erlbaum.

Tajfel, H. (1982). Social Psychology of Intergroup relations. Annual Review of Psychology, 33, 1-39. http://dx.doi.org/10.1146/annurev.ps.33.020182.000245

Tajfel, H. (1984). Grupos humanos y categorias sociales. Barcelona: Editorial Herder. 
Tajfel, H. \& Turner, J. (2001). An integrative theory of intergroup conflict. En Abrams, D. \& M. Hogg (Eds.), Intergroup relations: essential readings. Philadelphia: Psychology Press.

Van Vugt, M. \& Hart, C. (2004). Social Identity as Social Glue: The Origins of Group Loyalty. Journal of Personality and Social Psychology, 86, 585-598. http://dx.doi.org/10.1037/00223514.86.4.585

Vignoles, V. L., Regalia, C., Manzi, C., Golledge, J. \& Scabini, E. (2006). Beyond Self- Esteem: Influence of Multiple Motives on Identity Construction. Journal of Personality and Social Psychology, 90(2), 308-333. http://dx.doi.org/10.1037/00223514.90.2.308

Recibido el 01 de julio de 2014 Aceptado el 30 de septiembre de 2015 